\title{
A Model for Mobile Payment in Ubiquitous Commerce
}

\author{
Alex Roehrs ${ }^{1}$, Cristiano André da Costa ${ }^{1}$, Jorge Luis Victória Barbosa ${ }^{1}$ \\ ${ }^{1}$ Programa de Pós-Graduação em Computação Aplicada (PIPCA) \\ Universidade do Vale do Rio dos Sinos (UNISINOS) \\ Av. Unisinos, 950 - B. Cristo Rei / CEP 93.022-000 - São Leopoldo - RS - Brazil \\ alexroehrs@gmail.com, \{cac, jbarbosa\}@unisinos.br
}

\begin{abstract}
This paper aims at presenting an architectural model proposal for a novel Mobile Payment System, called 4iPay. This work considers the following premises: independence of device, location, carrier and cardholder to meet the needs of executing payment transactions in ubiquitous commerce. Our proposal considers the convergence of concepts of ubiquity, unity, universality and unison to form the proposed model. This article describes the model, implementation, preliminary assessments and requirements for mobile payment in ubiquitous environments. We developed a prototype of 4iPay using Android smartphones. Our protoype was evaluated in three diferente scenarious.
\end{abstract}

\section{Introdução}

Com o avanço da tecnologia da informação, o comércio também está evoluindo do atual eletroniccommerce (e-commerce) para o ubiquitous-commerce (u-commerce). Por definição, num ambiente ubíquo podem ser encontrados diversos tipos de dispositivos, dentre eles celular, televisor, tablet, e outros aparelhos que estejam ao alcance do usuário [12]. Assim, $u$ commerce é uma solução universal para todo tipo de necessidade de comércio que possa surgir [14]. De acordo com [15], quatro características definem ucommerce:

- Ubiquidade: usuários acessam a partir de qualquer lugar, a qualquer momento;

- Unicidade: usuários identificados de forma unívoca;

- Universalidade: dispositivos universalmente utilizáveis e multifuncionais;

- Unissonância: dados são integrados e consistentes em diferentes aplicativos.
Para prestar o serviço de cartão de crédito, um comerciante deve possuir conta em banco, alugar um terminal POS (Point of Sale), prever uma taxa de carregamento e outra taxa de manutenção mensal do POS. Com o pagamento via celular, o lojista precisa usar apenas um aparelho e uma conexão móvel de dados. Já com o micro-pagamento, é possível realizar transações diretamente entre comprador e vendedor. Os cartões de crédito não se destinam às relações interpessoais, que são feitas com dinheiro ou cheque. Com o uso de aparelhos celulares este tipo de transação se torna uma opção viável. Além disso, com o serviço móvel de pagamento, o usuário não necessita pagar taxas adicionais, já que o aparelho celular pode ser o seu próprio [13].

Desta forma, a motivação para este trabalho consiste em propor um modelo de arquitetura de pagamento móvel com características de fato ubíquas, ou seja, compreendendo as quatro principais premissas de um sistema ubíquo, composto pelo atendimento a ubiquidade, unicidade, universalidade e unissonância [15], com vistas a suportar pagamentos móveis em ambientes de comércio ubíquo. Embora o tradicional comércio eletrônico (e-commerce) seja um modelo popular de transações de pagamentos, ainda existem muitos aspectos que necessitam ser melhorados, sendo o pagamento móvel um dos gargalos para a adoção e desenvolvimento do comércio ubíquo [12].

O presente trabalho, denominado 4iPay, consiste em uma proposta de arquitetura de sistema de pagamento móvel para ambientes de comércio ubíquo e tem por objetivo propor uma solução diferenciada em relação ao tratamento dos requisitos fundamentais do $\mathrm{u}-$ commerce, citados por [15]. Como escopo do trabalho serão detalhados aspectos e propostas, levando em consideração, nas transações comerciais eletrônicas de transferência de fundos, questões como independência de dispositivo, de operadora, de administradora, de posição física e em relação à segurança e confiabilidade da aplicação. Neste interim, é importante destacar que, o modelo proposto visa não 
apenas atender ao pagamento móvel via aparelho celular, que é fundamental para o u-commerce, mas também reunir diferentes tecnologias num barramento de serviços para incorporar outros tipos de comércio eletrônico que possam envolver qualquer tipo de dispositivo com capacidade computacional e compor assim as quatro características do comércio ubíquo.

O artigo está organizado em 6 seções. A seção 2 descreve os trabalhos relacionados. $\mathrm{O}$ modelo proposto é detalhado na seção 3, incluindo a proposta de arquitetura. Na seção 4 são descritos os cenários de utilização. A seção 5 apresenta as avaliações realizadas e possíveis trabalhos futuros. Por fim, a seção 6 apresenta as conclusões.

\section{Trabalhos relacionados}

Para o desenvolvimento desta proposta foram analisados cinco sistemas que possuem características similares e compatíveis com as funcionalidades inerentes ao comércio ubíquo. Os modelos escolhidos foram determinados em função de características particulares relevantes ao comércio ubíquo, como a possibilidade de efetivar transações locais, à distância ou sensíveis ao contexto do usuário, níveis suportados de segurança e gerenciamento das transações, além de características subjetivas, como o nível de inovação quanto às tecnologias empregadas em relação aos formatos tradicionais de pagamento móvel. Os trabalhos analisados, incluindo o modelo proposto, levam em consideração as classificações [5] e [15], propondo uma atualização e congregação da classificação dos modelos, apresentada na Figura 1, onde observa-se que os modelos de pagamento móvel dividem-se em quatro grupos, com subdivisões: quanto ao modelo de negócio, quanto aos dispositivos, quanto à localização e quanto à segurança e confiabilidade.

$\mathrm{O}$ primeiro sistema analisado tem o nome de UbiPay [9]. Este sistema tem por principal objetivo minimizar os custos em pagamentos móveis, oferecendo uma gama de modos de interação e escolhendo o de mínimo valor. O objetivo é fazer com que pagar, seja como a respiração: algo que fazemos inconscientemente e sentimos apenas quando exercemos de forma além do habitual, ou seja, que aconteça a transferência de valores de forma transparente para o usuário.

A seguir foi analisado o sistema denominado mFerio [2]. Este sistema foi concebido e avaliado tendo como escopo a utilização da tecnologia NFC (Near Field Communication). O sistema mFerio é baseado em solicitação de pagamento móvel P2P (ponto-a-ponto), ou também denominado $p$-commerce. Em suma, consiste no comércio realizado por proximidade dos dispositivos envolvidos na transação de transferência de fundos; se destina a substituir as transações baseadas em dinheiro.

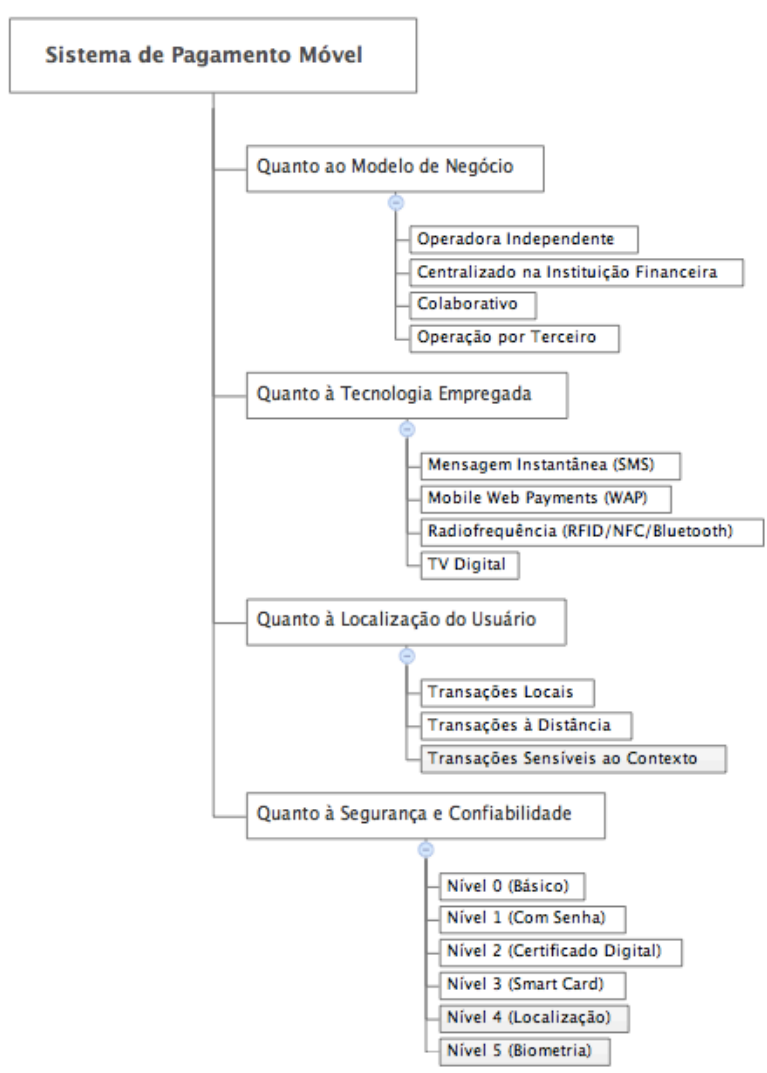

Figura 1. Modelos de Sistema de Pagamento

Outro sistema analisado possui o nome U-PR [8]. Este sistema tem por objetivo principal propor um regime de pagamento para comércio ubíquo promovendo a uniformidade, segurança e privacidade nas transações eletrônicas com dispositivos móveis. Tem como elemento fundamental da sua arquitetura e modelo de negócio, o suporte e proteção à privacidade.

O próximo sistema analisado tem o nome de SmartRestaurant [10]. Este sistema é um modelo de aplicação que permite aos clientes utilizarem dispositivos móveis para encomendar e pagar refeições a partir de um restaurante nas proximidades do campus da Universidade de Oulu, Finlândia. Além disso, o sistema é capaz de colaborar com a administração do restaurante, na medida que permite ajustar as vendas, verificar a capacidade de produção e ter conhecimento prévio de encomendas futuras.

Por fim, completando o conjunto de sistemas relacionados, foi analisado o sistema denominado fairCASH que, segundo [6], deve ser visto como um conjunto de tecnologias, coletivamente servindo ao 
apoio do ciclo de vida de objetos financeiros (tokens) anônimos e intransferíveis, denominados como eCoins, incluindo todas as fases: criação, manipulação, distribuição e compensação para evitar cópias ilegais.

O fairCASH é uma solução centrada em serviços ICT (Internet Communication Technology) que tem por objetivo operar um conjunto de ações, procedimentos, políticas, propriedades e ferramentas numa arquitetura distribuída de eWallets (digital wallet, ou seja, carteira digital), utilizadas para realizar os pagamentos. Em outras palavras o modelo fornece as principais tecnologias necessárias para construir um sistema de dinheiro digital (Digital Cash), o mais próximo possível ao seu homólogo físico bem conhecido.

\section{O Modelo 4iPay}

Nesta seção é apresentada a descrição do modelo proposto. O nome 4iPay dado ao modelo de sistema tem o objetivo de ilustrar seu acrônimo obtido da seguinte forma:

- 4 - significando suporte às quatro premissas da computação ubíqua: ubiquidade, unicidade, universalidade e unissonância, e uma paronomásia com a preposição da língua inglesa "for", em português "para";

- $\quad \mathrm{i}$ - significando a independência de dispositivo, de operadora, de administradora e de posição física;

- $\quad$ Pay - significando o objetivo principal do modelo que é o suporte ao pagamento móvel de transações eletrônicas de fundos (TEF), do inglês electronic funds transfer (EFT) [7].

A Figura 2 apresenta uma visão macro da arquitetura, na qual constam as divisões das camadas cliente e servidor utilizadas no sistema. A área pontilhada exibe a visão do escopo de atuação da aplicação, formada basicamente por uma camada servidora e aplicações cliente adaptadas a cada tipo diferente de dispositivo utilizado pelo usuário final. Nesta ilustração é possível observar que a aplicação cliente do comprador tem a possibilidade de se comunicar tanto com o servidor quanto diretamente com a aplicação cliente do dispositivo que está próximo, utilizando para tanto a tecnologia NFC, diferenciando assim uma transação local e a distância. Caso seja uma transação que envolva uma instituição financeira, a qual pode ser representada por um banco ou administradora de cartões, o servidor é responsável por realizar a comunicação com esta e efetivar a transação.
Já a aplicação cliente pode ser tanto utilizada pelo comprador quanto pelo vendedor. É possível também realizar uma transferência financeira local entre um dispositivo móvel e uma máquina fixa, como no caso da compra de um refrigerante ou pagamento de ticket de metrô. Também é possível observar na ilustração que tanto o comprador quanto o vendedor possuem uma comunicação com o servidor, com o objetivo de efetivar e consolidar as transferências realizadas de forma local entre dispositivos.

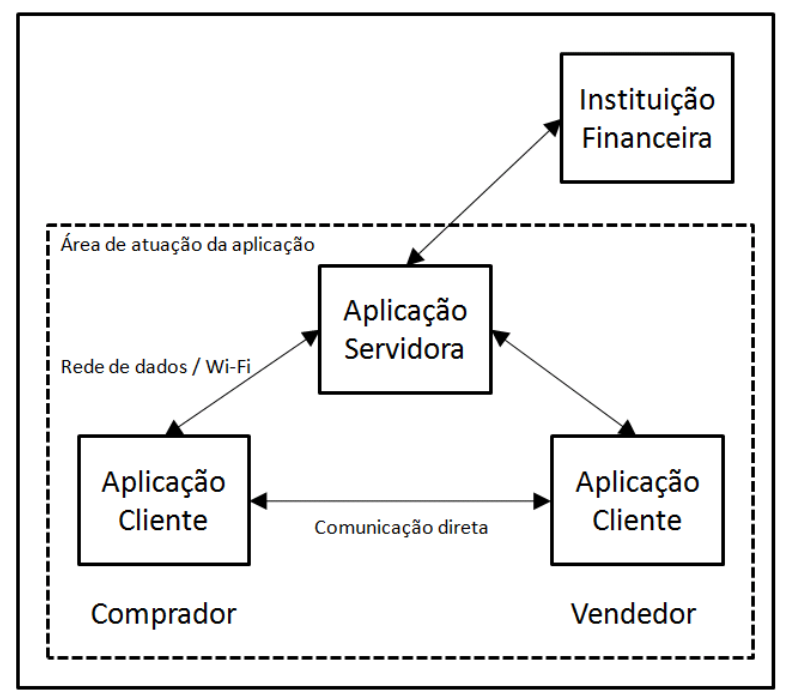

Figura 2. Visão Geral da Arquitetura

A Figura 3 mostra uma visão do diagrama de componentes do modelo $4 \mathrm{iPay}$, onde consta a camada representada pelos dispositivos possíveis de utilização, a camada de entrada na aplicação, formada por um gateway ou webservices de recebimentos das requisições, um barramento de serviços, responsável pelo armazenamento e distribuição das requisições, e os diversos slots de serviços possíveis de serem invocados. Além disso, o modelo proposto prevê um site de gerenciamento e monitoramento das transações. A infraestrutura do sistema 4iPay é baseada na arquitetura ESB (Enterprise Service Bus) [4], com o objetivo de fornecer uma categoria de serviços independentes e um barramento de serviços complexos baseados no processamento de mensagens de transações eletrônicas de fundos. Conforme pode ser observado na Figura 3, o modelo 4iPay está dividido em camadas verticais, onde, da esquerda para a direita, na primeira camada, se pode observar a multiplicidade de dispositivos suportados. A segunda camada tem por objetivo demonstrar o suporte a múltiplos protocolos de recepção e resposta aos dispositivos integrados, como SOAP (Simple Object Access Protocol), REST (Representational State Transfer), SMS (Simple 
Message System), JMS (Java Message Service) e JCA (Java Connector Architecture). A terceira camada diz respeito ao barramento ESB, responsável pela integração e gerenciamento de todo o ambiente. A quarta camada tem por objetivo demonstrar as interconexões com as instituições financeiras, através da construção e invocação de regras de negócio de forma transparente, visto que o ESB pode fazer uso de diferentes plataformas de desenvolvimento e linguagens [4], tornando transparente ao sistema a interligação com diferentes sistemas das administradoras.

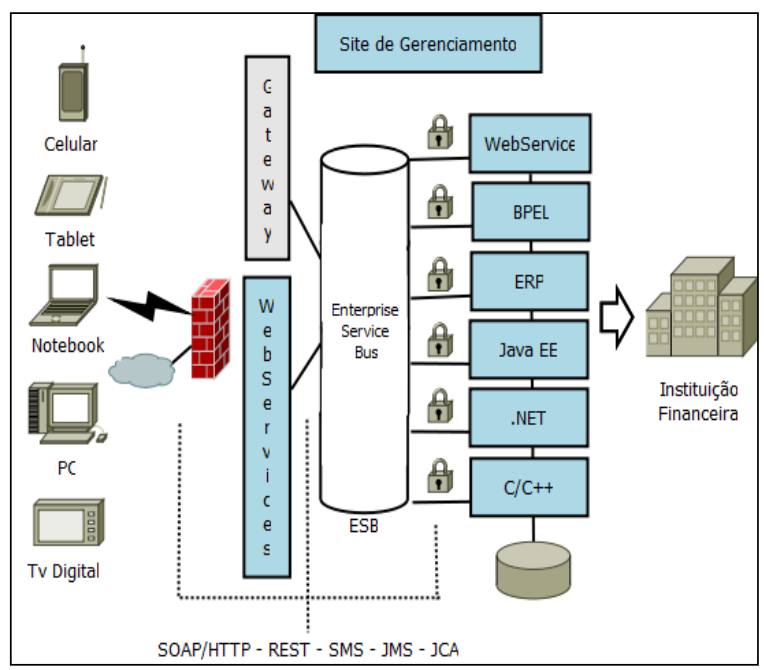

Figura 3. Diagrama de Componentes do 4iPay

A infraestrutura do sistema 4iPay é baseada na arquitetura ESB, com o objetivo de prover uma categoria de serviços independentes de plataforma de invocação, fornecendo uma base de serviços complexos baseados no processamento de mensagens de transações eletrônicas de fundos. Este sistema tem o objetivo também de fornecer uma camada de abstração da implementação do sistema, de forma a permitir uma integração das mensagens transparentes segundo a arquitetura, fornecendo as características necessárias a uma arquitetura orientada a serviços (SOA - Service Oriented Architecture) [11]. Outro objetivo desta arquitetura é possibilitar padrões flexíveis no transporte e interpretação das mensagens trocadas com os dispositivos, suportando diferentes padrões e aparelhos, removendo assim o acoplamento entre o serviço invocado e o protocolo de transporte.

\subsection{Arquitetura Proposta}

A Figura 4 apresenta o detalhamento da arquitetura do 4iPay. Nesta figura são apresentados os componentes da arquitetura proposta, a qual é formada por um barramento de serviços na camada servidora, subdividida em núcleo central, responsável pelos conversores, parseadores, gerenciamento de controle e gerenciador de exceções ou falhas, adaptadores para suporte a diferentes protocolos e um framework de suporte ao reaproveitamento dos diversos componentes da arquitetura. $\mathrm{O}$ framework da aplicação promove a distribuição das camadas de visão, controle, negócio e persistência, colaborando para a reutilização dos componentes construídos.

Os adaptadores se fazem necessários em função da proposição de independência de plataforma, bem como suporte a diferentes protocolos de comunicação, tanto a nível de interface com a camada cliente, quanto interface com as operadoras e administradoras financeiras. Também constam da infraestrutura proposta um repositório de serviços, um site de gerenciamento e uma camada de componentes de segurança, utilizados por toda a aplicação. A descrição da utilização dos componentes de suporte ao barramento de serviços é detalhada no item a seguir.

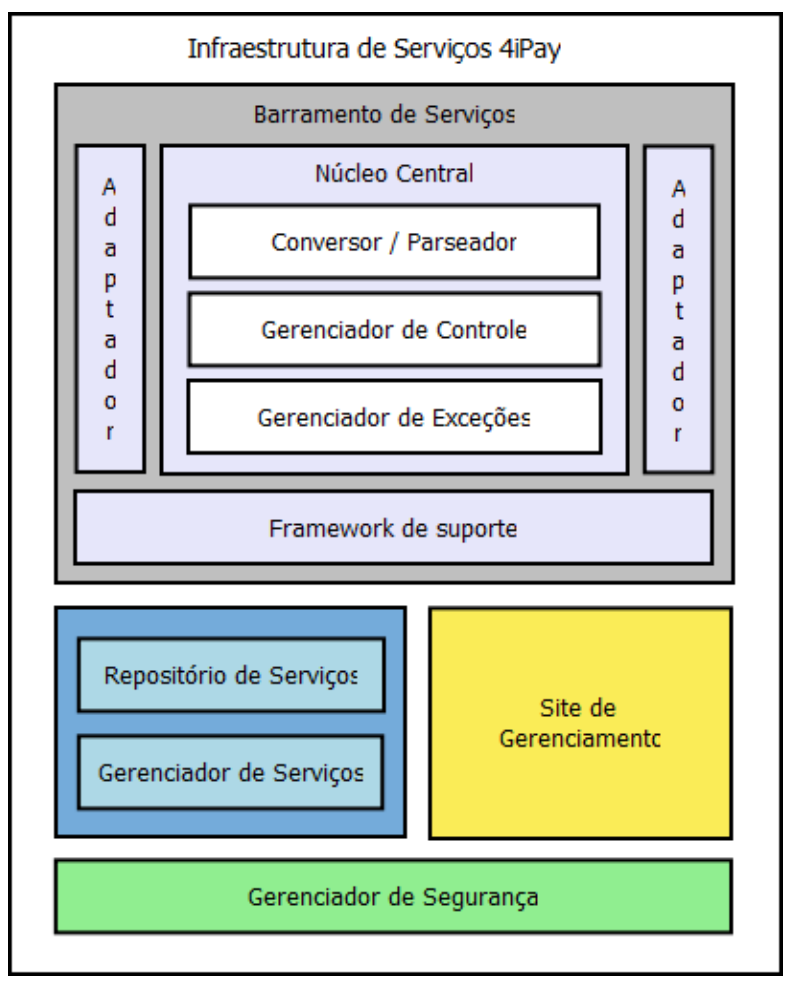

Figura 4. Diagrama Infraestrutura de Serviços

3.1.1. Utilização de ESB. A proposta de incorporar um barramento de serviços (Enterprise Service Bus) advém da utilização desta arquitetura de forma a abstrair tanto o formato dos dados quanto dos protocolos que estarão envolvidos, facilitando a 
integração entre sistemas heterogêneos, já que impõem um contrato específico para a troca de mensagem entre os sistemas participantes. Com o ESB o sistema está apto a utilizar componentes como [4]:

- Invocação: Componente responsável por prover suporte a protocolos de maneira síncrona e assíncrona;

- Roteamento: Componente responsável por enviar as informações para determinados destinos, de forma estática ou dinâmica;

- Transformação: Componente responsável por prover a transformação de protocolos, promovendo a interdependência de integração;

- Mensageria: Componente responsável por prover o tratamento, processamento e reenvio de mensagens. Este item tem especial importância nos casos em que se faz necessário a resubmissão da transação, caso por exemplo de algum erro ou serviço que ao ter sido invocado estivesse fora do ar. Assim, a transação não é descartada e pode ser reaproveitada;

- Orquestração: Componentes relacionados a processos complexos e BPMN/BPEL integrados à invocação de WebServices [4].

\section{Cenários}

Nesta seção são apresentados alguns cenários de casos de teste possíveis para avaliar a utilização do modelo, os quais compõem os principais aspectos que o sistema tem por objetivo atender.

Na seção seguinte é descrita a execução e avaliação desses três cenários.

\subsection{Cenário 1 - Pagamento Local Sensível ao Contexto}

Cenário que pretende demonstrar a utilização do modelo em uma transação de pagamento local, mas entre um dispositivo móvel e uma máquina fixa, com um pagamento por aproximação. Este é o cenário onde o usuário possui um aparelho com suporte a tecnologia de aproximação (NFC) e deseja realizar o pagamento em algum terminal que tenha suporte a este tipo de tecnologia. Um exemplo de uso deste caso é o pagamento de ticket de metrô, ou então a compra de um refrigerante em máquinas que suportam esta tecnologia.

Além do pagamento através da tecnologia de aproximação, o modelo suporta um nível de segurança de acordo com o contexto do usuário. Como exemplo, o usuário pode, em um determinado contexto identificado, autorizar o pagamento com nível de segurança menor ou maior de acordo com suas preferências. O contexto, neste caso, é determinado por sua localização geográfica, utilizando para tanto o GPS do aparelho.

Como exemplo, o usuário pode autorizar de forma automática o pagamento do estacionamento da universidade, onde ele está frequentemente e tem configurado nas suas preferências pessoais, somente aproximando seu aparelho celular no terminal de validação, que pode estar localizado na própria cancela de saída.

Outra forma simples de realizar esta operação, sem a utilização do recurso de GPS, é utilizar pagamentos pré-configurados, com o nome de pagamentos favoritos para os pagamentos do dia-a-dia, tais como, metrô, estacionamento, refrigerante de máquina, etc.

\subsection{Cenário 2 - Pagamento entre Dispositivos}

Este cenário pretende demonstrar a utilização do modelo em uma transação de pagamento local, todavia realizando uma transferência entre dispositivos móveis, onde o comprador e o vendedor estão próximos, utilizando cada um seu aparelho. Nesta situação o sistema tem a possibilidade de efetivar uma transação sem passar por um servidor no momento exato que está ocorrendo a transação.

Neste caso os meios de conexão podem ser a tecnologia de aproximação (NFC), Bluetooth ou mesmo uma rede sem fio (Wireless) se for o caso. Esta situação pode ocorrer por basicamente dois motivos: quando não há conexão remota dos aparelhos com qualquer rede de dados ou quando não há necessidade de efetivar a transação naquele exato instante. Um exemplo é o pagamento de uma corrida de táxi no subsolo de um shopping center.

Provavelmente o sinal da rede de dados da operadora estará indisponível ou mesmo que esteja disponível o taxista pode optar por efetivar todas as transações do dia em uma transação única ao final do dia. Neste caso o sistema deverá prever a efetivação em lote de transações.

\subsection{Cenário 3 - Pagamento Remoto}

Cenário que pretende demonstrar a utilização do modelo em um pagamento remoto, ou seja, com o comprador e o vendedor estando distantes um do outro. Este cenário faz uso de conexões de longa distância, tais como a rede de dados da operadora de telefonia, uma rede sem fio ou mesmo mensagens SMS. Nesta situação o comprador e o vendedor estão distantes. Exemplos são deliverys, como o pagamento de uma pizza, onde o cliente está em casa e precisa efetivar o 
pagamento antes da entrega. Neste caso, o vendedor irá requisitar um pagamento, o qual será efetivado remotamente com a autorização do comprador. Outra situação que poderá acontecer é autorização por terceiro. Um exemplo deste caso é o filho que se dirige a uma padaria de bairro para comprar pão, mas não tem créditos para efetivar o pagamento com o seu aparelho. Assim, o vendedor remete a requisição para o número da mãe que já está aguardando para efetivar o pagamento pelo aparelho dela.

\section{Avaliação}

Para a avaliação do trabalho foram utilizados os três cenários descritos e empregado para tanto os recursos da plataforma de desenvolvimento Google Android, com a IDE Eclipse e os respectivos plug-ins necessários, na versão 2.3.3, codinome Gingerbread, a qual possui suporte à tecnologia NFC [1]. Como estudo de caso utilizou-se a implementação base desenvolvida para um outro projeto em desenvolvimento pelo nosso grupo, denominado Mingle [3]. O projeto Mingle tem por objetivo gerenciar a interação ubíqua numa rede social dinâmica, com o mínimo de infraestrutura, formada pelas pessoas que estão fisicamente presentes em determinados locais, sendo então o modelo 4iPay aplicado sobre esta plataforma. O principal resultado consiste no desenvolvimento de um sistema de interação ubíqua, funcional, e que possa ser aplicado a diversos cenários reais, tanto com exploração comercial como puramente social [3]. O modelo 4iPay, neste caso, atua como um módulo de serviço comercializável dentro da infraestrutura disponibilizada pela rede Mingle.

No cenário 1 a transação ocorre entre o dispositivo que possui suporte à tecnologia NFC, representando o aparelho do usuário pagador e uma tag, ou seja um aparelho fixo também compatível, que faz o papel de receptor da transação de pagamento. Ainda no cenário 1, o pagamento pode ocorrer com um nível de segurança diferenciado no caso do pagamento decorrer em um determinado local de acordo com as preferências pré-configuradas do usuário. Assim, o usuário pode, por exemplo, autorizar pagamentos de forma automática dependendo do contexto onde ele esteja localizado. No cenário 2 as transações ocorrem entre dispositivos, seja por conexão wireless, bluetooth ou NFC, representando transações locais entre aparelhos que estejam localizados próximos um do outro. Neste caso a transferência de valores pode ocorrer de forma direta de aparelho para aparelho, representando uma transação P2P, mas com a consolidação efetiva da transação ocorrendo por intermédio do sistema de controle localizado na camada servidora, a qual é responsável, tanto pela efetivação do débito e crédito nas contas dos usuários, quanto pelo gerenciamento e tratamento das pendências ou erros que possam ocorrer nas transações. O cenário 3 demonstra o pagamento à distância. A Figura 5 demonstra os meios de transferência dos dados de pagamento que o sistema 4iPay suporta, onde se pode observar, na ordem, conexão wireless, rede de dados da operadora, bluetooth, NFC e via SMS. Nesta figura também é possível observar que apenas a conexão NFC está ligada.

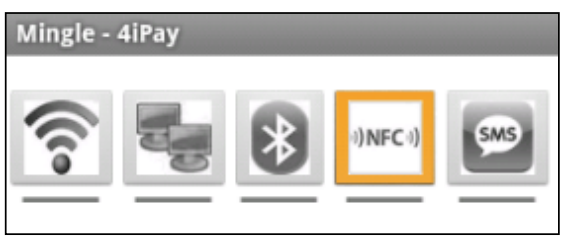

Figura 5. Possibilidades de transmissão

A seguir são apresentados os passos de como ocorre um pagamento utilizando a aplicação do modelo 4iPay sobre a plataforma Mingle como estudo de caso. A Figura 6 demonstra alguns screenshots dos cenários avaliados. Para o primeiro cenário, que consiste no pagamento por aproximação em relação à tag, a qual representa o dispositivo fixo de recepção da transação, a plataforma Android disponibiliza uma API denominada NFC Data Exchange Format (NDEF) para a leitura de tags [1] [16] . O segundo cenário representou a transferência eletrônica de fundos entre dois dispositivos que estão próximos, utilizando para tanto a tecnologia NFC para um pagamento local e de forma direta $\mathrm{P} 2 \mathrm{P}$. O terceiro cenário constituiu um pagamento remoto, através de tecnologia $\mathrm{WiFi}$.

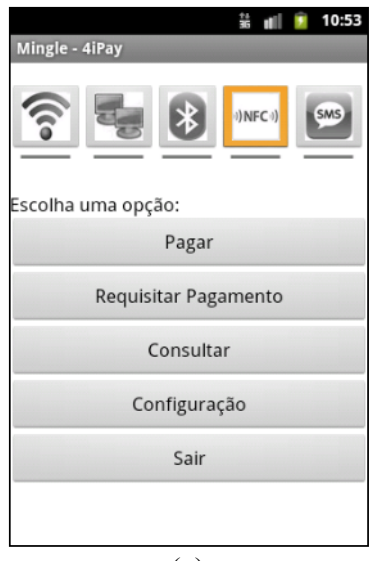

(a)

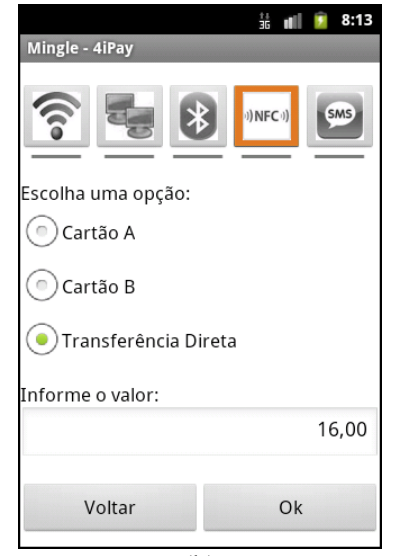

(b) 


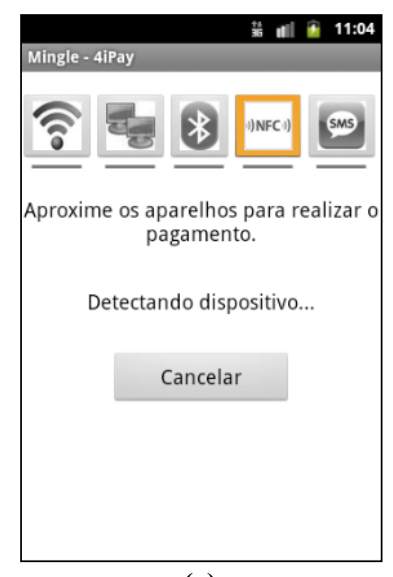

(c)

Figura 6. Interfaces da Aplicação

A Figura 6 (a) apresenta a primeira tela, que mostra um cenário onde o usuário pode optar entre realizar um pagamento, requerer o pagamento de outro usuário um exemplo é o caso de um vendedor requerendo um pagamento do cliente, utilizado para representar o terceiro cenário - , consultar pagamentos efetuados e configurar a aplicação com preferências particulares. Para a avaliação da autorização remota, o recurso relevante para este tipo de transação é a notificação do usuário que há um pagamento para autorizar através da barra de notificações utilizada pelo sistema Android [1]. Através desta barra a aplicação possui condições de notificar o usuário sobre qualquer evento. Para a autorização à distância, este recurso é especialmente importante, já que consegue notificar o usuário de forma leve e discreta sobre uma transação pendente de autorização, por exemplo. Na Figura 6 (b), segunda tela, são exibidas as opções relacionadas com a determinação da forma de pagamento, caso o usuário tenha escolhido a primeira opção na tela anterior. A Figura 6 (c), terceira tela, demonstra a realização da transferência entre dispositivos que estão próximos (cenário 2), configurando um pagamento do tipo local. Para tanto, o sistema tem a capacidade de detectar que apenas o suporte a NFC está ligado na aplicação, como se observa nos ícones superiores de cada tela. Desta forma, a aplicação consegue realizar o pagamento com pouca e transparente interação com o usuário. Por fim, a Figura 6 (d) apresenta o resultado final da transação.

\subsection{Comparação com outros sistemas}

A Tabela 1 apresenta um comparativo das principais características dos modelos analisados. $\mathrm{O}$ comparativo inicia com a análise da arquitetura de cada modelo. São analisados os modelos quanto à possibilidade de atender transações P2P e cliente- servidor, ou seja, se possuem a capacidade de atender requisições locais e à distância.

Tabela 1. Comparação entre Modelos Relacionados

\begin{tabular}{|c|c|c|c|c|c|c|c|}
\hline \multicolumn{2}{|c|}{$\begin{array}{c}\text { Modelo / } \\
\text { Característica }\end{array}$} & UbiPay & mFerio & U-PR & $\begin{array}{c}\text { Smart } \\
\text { Restaurant }\end{array}$ & fairCASH & 4iPay \\
\hline \multicolumn{2}{|c|}{ Arquitetura } & P2P & P2P & $\begin{array}{l}\text { Cliente- } \\
\text { Servidor e }\end{array}$ & Cliente- & $\begin{array}{l}\text { Cliente- } \\
\text { Servidor e }\end{array}$ & \begin{tabular}{|c|} 
Cliente- \\
Servidor e P2P
\end{tabular} \\
\hline \multirow{8}{*}{ 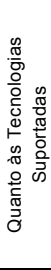 } & SMS & Não & Não & Não & Não & Não & Sim \\
\hline & WAP & Não & Não & Não & Sim & Não & Sim \\
\hline & NFC & Sim & Sim & Sim & Não & Sim & Sim \\
\hline & Bluetooth & Sim & Sim & Sim & Não & Sim & Sim \\
\hline & Ethernet & Não & Não & Não & Sim & Sim & Sim \\
\hline & Wi-Fi & Sim & Sim & Sim & Sim & Sim & Sim \\
\hline & USB & Não & Não & Sim & Sim & Sim & Sim \\
\hline & $\begin{array}{c}\text { TV } \\
\text { Digital }\end{array}$ & Não & Não & Não & Sim & Sim & Sim \\
\hline \multirow{3}{*}{ 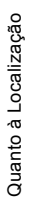 } & Local & Sim & Sim & Sim & Sim & Sim & Sim \\
\hline & $\begin{array}{c}\dot{A} \\
\text { distância }\end{array}$ & Sim & Não & Não & Sim & Sim & Sim \\
\hline & $\begin{array}{l}\text { Sensivel } \\
\text { ao } \\
\text { contexto }\end{array}$ & Sim & Não & Não & Sim & Não & Sim \\
\hline \multirow{6}{*}{ 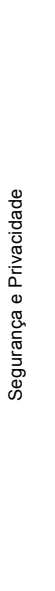 } & $\begin{array}{l}\text { Distrib. da } \\
\text { Informação }\end{array}$ & Regular & Regular & Alta & Baixa & Alta & Alta \\
\hline & $\begin{array}{c}\text { Nivel de } \\
\text { Segurança da } \\
\text { Informação }\end{array}$ & \begin{tabular}{|} 
Proteção \\
regular \\
(Niveis \\
0,1 )
\end{tabular} & $\begin{array}{l}\text { Proteção } \\
\text { regular } \\
\text { (Niveis } \\
0,1,5 \text { ) }\end{array}$ & $\begin{array}{l}\text { Alta proteção } \\
\text { (Niveis } 1,2,5 \text { ) }\end{array}$ & $\begin{array}{c}\text { Baixa } \\
\text { proteção } \\
\text { (Níveis } 0,1)\end{array}$ & $\begin{array}{l}\text { Alta } \\
\text { proteção } \\
\text { (Niveis } \\
1,2,3,4)\end{array}$ & $\begin{array}{l}\text { Alta proteção } \\
\text { (Níveis } 0,1,4,5 \text { ) }\end{array}$ \\
\hline & $\begin{array}{l}\text { Criptografia } \\
\text { dos dados }\end{array}$ & \begin{tabular}{|c|} 
Dos \\
dados \\
armaze- \\
nados e \\
transmiti- \\
dos
\end{tabular} & $\begin{array}{l}\text { Dos dados } \\
\text { armazena- } \\
\text { dos e } \\
\text { transmiti- } \\
\text { dos }\end{array}$ & \begin{tabular}{|c} 
Dos dados \\
armazena-dos \\
e transmiti-dos
\end{tabular} & $\begin{array}{c}\text { Dos dados } \\
\text { transmiti- } \\
\text { dos }\end{array}$ & $\begin{array}{l}\text { Dos dados } \\
\text { armazena- } \\
\text { dos e } \\
\text { transmiti- } \\
\text { dos }\end{array}$ & $\begin{array}{l}\text { Dos dados } \\
\text { armazenados e } \\
\text { transmiti-dos }\end{array}$ \\
\hline & $\begin{array}{l}\text { Criptografia } \\
\text { mensagens }\end{array}$ & Sim & Sim & Sim & Não & Sim & Sim \\
\hline & $\begin{array}{l}\text { Autenticação } \\
\text { rápida }\end{array}$ & Não & $\begin{array}{l}\text { Sim, por } \\
\text { biometria } \\
\text { ou senha } \\
\text { gráfica }\end{array}$ & Não & Não & Não & $\begin{array}{l}\text { Sim, por } \\
\text { biometria } \\
\text { ou senha } \\
\text { gráfica }\end{array}$ \\
\hline & $\begin{array}{c}\text { Autenticação } \\
\text { automática }\end{array}$ & Sim & Não & Não & Não & Não & Sim \\
\hline \multicolumn{2}{|c|}{$\begin{array}{c}\text { Site de } \\
\text { Gerenciamento }\end{array}$} & Não & Não & Sim & Sim & Não & Sim \\
\hline
\end{tabular}

Em comparação com o sistema proposto, o sistema UbiPay [9] tem um foco mais concentrado no micropagamento, utilizando apenas a tecnologia NFC para realizar transferências eletrônicas de fundos de diversas formas, mas somente entre dispositivos que utilizam a mesma tecnologia. Assim, como o sistema anterior, o sistema mFerio se concentra exclusivamente na tecnologia NFC para realizar os pagamentos, mas com a diferença de inovar utilizando a tecnologia de biometria para autenticar e autorizar as transações. O terceiro sistema analisado, U-PR também utiliza apenas a tecnologia P2P-Commerce, ou seja, o comércio ubíquo por aproximação de dispositivos [15], mas com um foco em segurança e privacidade, propondo um sistema com diversos controles de segurança, mas de forma transparente para os 
participantes. Já o sistema SmartRestaturant consiste em um modelo que baseia-se somente na tecnologia de WebService, não permitindo o pagamento entre dispositivos, mas que pode ser acessado por smartphones, tablets ou qualquer computador com acesso a Internet. Além disso, este sistema possui o diferencial de permitir que os usuários façam encomendas, já que se trata de um sistema para restaurantes, estendendo assim a funcionalidade de pagamento. Em relação ao modelo fairCASH [6], o modelo se diferencia em contemplar, além de muitas características já existentes em outros modelos, transações efetuadas com sensibilidade ao contexto, possibilidade de utilizar diversos níveis de segurança, inclusive com agregação, como biometria e localização; utilização de qualquer tecnologia de comunicação móvel disponível, independência de dispositivo e de localização dos participantes das transações.

\section{Conclusão}

O pagamento móvel possui um grande potencial, sendo que o modelo de pagamento móvel do futuro deverá ser de qualquer tipo, realizado por qualquer um, com qualquer aparelho, em qualquer lugar, de qualquer valor e para qualquer tipo de negócio. É neste âmbito que o modelo 4iPay se insere, propondo uma solução independente de dispositivo, localização, operadora e instituição financeira para a efetivação de transações eletrônicas de fundos.

Para tanto, o modelo procura atender cenários de utilização de forma tanto local quanto à distância de transações com diferentes dispositivos, deixando transparente ao usuário questões como o tipo de conexão utilizada. Como trabalhos futuros, pretende-se adicionar suporte a diferentes plataformas de desenvolvimento, bem como portar a aplicação para diferentes dispositivos móveis.

Outro foco do trabalho são as melhorias de implementação em termos de segurança da informação e otimização do desempenho da aplicação, fundamentais em um sistema de pagamento móvel. Também pretende-se pesquisar e avaliar outros cenários de utilização com diferentes dispositivos e protocolos de comunicação.

\section{Referências}

[1] Android. "Near Field Communication", API, 2011. Acessado em 10 de Março, 2011 em: http://developer.android.com/guide/topics/nfc/index.html

[2] Balan, R. K., Ramasubbu, N., Prakobphol, K., Christin, N., Hong, J. "mFerio: The Design and Evaluation of a Peer-
to-Peer Mobile Payment System." MobiSys'09, Kraków, Poland, June 22-25, 2009.

[3] Silva, B.; Costa, C. A.; Zaupa, D.; Freitas, G. “An Ontology-based Repository for a Spontaneous Social Network". CASoN 2011, Salamanca, Spain, October 19-21, 2011..

[4] Chappell, David A. "Enterprise Service Bus - Theory In Practice”. O'Reilly Media, Inc. First Edition, Gravenstein Highway North, Sebastopol, CA 95472, USA, 2004.

[5] Karnouskos, S. and Fokus, F. "Mobile payment: a journey through existing procedures and standardization initiatives". Communications Surveys \& Tutorials, IEEE, 6, n. 4. 44-66, 2004, DOI: 10.1109/COMST.2004.5342298.

[6] Kreft, H. "fairCASH based on Loss resistant Teleportation”, Kiel, Germany, 2010.

[7] Lassila, D., Lapine, K., Andress, R., McCullough, B., Pilecki, P., Weisblat, H. "Electronic Funds Transfer. Banking Law". Acessado em 10 de Abril de 2011 em: http://litigationessentials.lexisnexis.com, 2010.

[8] Lee, K. J., Ju, J., Jeong, J. “A Payment \& Receipt Business Model in U-Commerce Environment". Kyung Hee University of Business Administration. ICEC'06, Fredericton, Canada, ACM, August 14-16, 2006.

[9] Lehdonvirta, V., Soma, H., Hitoshi, I., Yamabe, T., Kimura, H., Nakajima, T. "UbiPay: Minimizing Transaction Costs with Smart Mobile Payments”. Mobility 2009, Sep 2-4, Nice, France, ACM, 2009.

[10] Lukkari, J., Korhonen, J., Ojala, T. "SmartRestaurant Mobile Payments in Context-Aware Environment". ICEC'04, Sixth International Conference on Electronic Commerce, 2004.

[11] Papazoglou M. P.; Georgakopoulos, D. "Introduction: Service-oriented computing". Communications of the ACM, New York, v.46, n.10, p. 24-28, 2003.

[12] Qiang, Yan, and Tingjie, Lu. "A Study of Mobile Payment Mode in U-commerce. Economics and Management School". Beijing University of Posts and Telecommunications. Beijing, China, 2008.

[13] Tiwari, R. and Buse, S. "The Mobile Commerce Prospects: A strategic analysis of opportunities in the banking sector". Hamburg: Hamburg University Press, 2007.

[14] Watson, R., Pitt, L., Berthon, P., Zinkhan, G. "Ucommerce: expanding the universe of marketing". Vol. 30(4). Journal of the Academy of Marketing Science, 2002.

[15] Xin, C. "U-Commerce New Technologies and Issues. Wuhan University Guangdong University of Foreign Studies". 2nd International Conference on Power Electronics and Intelligent Transportation System, China, 2009.

[16] NFC. "The Near Field Communication (NFC) Forum", 2011. Acessado em 10 de Março, 2011 em: http://www.nfcforum.org/home 